\title{
Correction to Approximation with Restricted Spectra
}

Math. Zeitschr. 144, 289-297 (1975)

Charles K. Chui, Philip W. Smith and Joseph D. Ward

Department of Mathematics, Texas A \& M University, College Station, Texas 77843, USA

The purpose of this note is to correct the proof of Theorem 4 in [1]. The result in Theorem 4 is still correct. However, the application of Berberian's result in the proof is incorrect. In what follows we use the same notation as in [1].

Theorem 4. Let $C$ be a closed convex set of cardinality larger than one in the complex plane. Then any normal operator $N$ has a unique best approximant $P_{C}(N)$ from $\mathfrak{N}(C)$ if and only if

$$
\widehat{d}(\sigma(N), C)=d(s, C)
$$

for all $s \in \sigma(N)$. Furthermore,

$$
\hat{d}(\sigma(N), C)=\left\|N-P_{C}(N)\right\| .
$$

In order to prove the above theorem, we need the following lemmas.

Lemma 1. With the same notation as in Theorem 4, $\hat{d}(\sigma(N), C)=\left\|N-P_{C}(N)\right\|$. This result is a distance formula of Halmos (cf. reference [6] in [1]).

Since $\sigma(N)$ is compact, we need only consider a compact restriction $K$ of $C$. If $K$ is a line segment, the proof would follow similarly and hence we assume $K$ to be a compact convex body. Let $K_{\alpha}=\{x: d(x, K)=\alpha\}$ and $y$ an interior point of $K$. Let $r_{n, i}, i=1, \ldots, n+1\left(r_{n, n+1}=r_{n, 1}\right)$, be rays emanating from $y$ and terminated by $K_{\alpha}$ with each angle, $l_{i}$, between $r_{n, i}$ and $r_{n, i+1}$, equal to $2 \pi / n$. Let $x_{n, i}=r_{n, i} \cap K_{\alpha}$ and $z_{n, i}$ the arc on $K_{\alpha}$ subtended by the angle $l_{i}$ and the chord $y_{n, i}$ connecting the points $x_{n, i}$ and $x_{n, i+1}$. Finally, let $d_{n, i}=d\left(y_{n, k}, z_{n, k}\right)$.

Lemma 2. There is a sequence $\varepsilon_{n} \rightarrow 0$ such that $d_{n, k} \leqq \varepsilon_{n} / n, k=1, \ldots, n$.

To prove this lemma, consider the triangle with sides $r_{n, i}, r_{n, i+1}$ and $y_{n, i}$. Since $K_{\alpha}$ is a convex curve, there is a $\delta>0$ such that the two angles facing $r_{n, i}$ and $r_{n, i+1}$ are between $\delta$ and $\pi-\delta$. Thus, using the law of sines,

$$
\left|y_{n, i}\right| \leqq C_{1} \sin \frac{2 \pi}{n} \leqq C_{2} / n
$$


for some constant $C_{2}$ independent of $i$ and $n$. Now, using the fact that the radius of curvature for each point on $K_{\alpha}$ is at least $\alpha$, it is easy to see that

$$
d_{n, i} \leqq \varepsilon_{n}^{\prime}\left|y_{n, i}\right| \leqq \varepsilon_{n}^{\prime} C_{2} / n \equiv \varepsilon_{n} / n \text {. }
$$

Returning to Theorem 4 , let $x_{i} \in H_{i},\left\|x_{i}\right\|=1$, where $H_{i}$ is a reducing subspace of $N$ such that $\sigma\left(\left.N\right|_{H_{i}}\right)=z_{n, i}$. If $N^{\prime}$ is a best approximant to $N$, then

$$
\alpha \geqq\left\|\left(N-N^{\prime}\right) x_{i}\right\| \geqq\left|\left\langle\left(N-N^{\prime}\right) x_{i}, x_{i}\right\rangle\right| \geqq \alpha-\varepsilon_{n} / n .
$$

Hence, $\left(N-N^{\prime}\right) x_{i}=\left(\alpha-\delta_{n, i} / n\right) x_{i}+f$, where $0 \leqq \delta_{n, 1}, \ldots, \delta_{n, n} \leqq \delta_{n} \equiv \delta_{n}\left(\varepsilon_{n}, N^{\prime}\right) \rightarrow 0$, $\left\langle f, x_{i}\right\rangle=0$ and $\|f\| \leqq \delta_{n} / \sqrt{n}$. Thus, if $N_{1}$ and $N_{2}$ are any two best approximants to $N$ and $\|x\|=1$, then

$$
\begin{aligned}
\left\|\left(N_{1}-N_{2}\right) x\right\| & \equiv\left\|\sum_{i=1}^{n} \alpha_{i}\left(N_{1}-N_{2}\right) x_{i}\right\| \leqq \sum_{i=1}^{n}\left|\alpha_{i}\right|\left\|\left(N_{1}-N_{2}\right) x_{i}\right\| \\
& \leqq\left(\sum_{i=1}^{n}\left\|\left(N_{1}-N_{2}\right) x_{i}\right\|^{2}\right)^{1 / 2} \leqq\left[n\left(\eta_{n} / \sqrt{n}\right)^{2}\right]^{1 / 2}=\eta_{n} \rightarrow 0
\end{aligned}
$$

where $\eta_{n}$ comes from $\delta_{n}\left(\varepsilon_{n}, N_{1}\right)$ and $\delta_{n}\left(\varepsilon_{n}, N_{2}\right)$. Thus, $\left(N_{1}-N_{2}\right) x=0$ for all $x$, or $N_{1}=N_{2}$.

The proof of the converse is the same as in [1].

We also note that the reference to the Berberian embedding in the second line of the proof of Theorem 6 should be omitted.

Remark. We would like to thank Professor J.P. Williams for pointing out the error in [1].

\section{References}

1. Chui, C.K., Smith, P.W., Ward, J. D.: Approximation with restricted spectra. Math. Z. 144, 289-297 (1975) 